\title{
Recombination-Mediated Genetic Engineering of a Bacterial Artificial Chromosome Clone of Modified Vaccinia virus Ankara (MVA)
}

\author{
Matthew G. Cottingham*, Rikke F. Andersen, Alexandra J. Spencer, Saroj Saurya, Julie Furze, Adrian V. S. \\ Hill, Sarah C. Gilbert
}

Wellcome Trust Centre for Human Genetics and The Jenner Institute, University of Oxford, Oxford, United Kingdom

\begin{abstract}
The production, manipulation and rescue of a bacterial artificial chromosome clone of Vaccinia virus (VAC-BAC) in order to expedite construction of expression vectors and mutagenesis of the genome has been described (Domi \& Moss, 2002, PNAS 99 12415-20). The genomic BAC clone was 'rescued' back to infectious virus using a Fowlpox virus helper to supply transcriptional machinery. We apply here a similar approach to the attenuated strain Modified Vaccinia virus Ankara (MVA), now widely used as a safe non-replicating recombinant vaccine vector in mammals, including humans. Four apparently fulllength, rescuable clones were obtained, which had indistinguishable immunogenicity in mice. One clone was shotgun sequenced and found to be identical to the parent. We employed GalK recombination-mediated genetic engineering (recombineering) of MVA-BAC to delete five selected viral genes. Deletion of C12L, A44L, A46R or B7R did not significantly affect $C D 8^{+} T$ cell immunogenicity in BALB/c mice, but deletion of $B 15 R$ enhanced specific $C D 8^{+} T$ cell responses to one of two endogenous viral epitopes (from the E2 and F2 proteins), in accordance with published work (Staib et al., 2005, J. Gen. Virol. 86, 1997-2006). In addition, we found a higher frequency of triple-positive IFN- $\gamma$, TNF- $\alpha$ and IL-2 secreting E3-specific CD8+ T-cells 8 weeks after vaccination with MVA lacking B15R. Furthermore, a recombinant vaccine capable of inducing $\mathrm{CD}^{+} \mathrm{T}$ cells against an epitope from Plasmodium berghei was created using GalK counterselection to insert an antigen expression cassette lacking a tandem marker gene into the traditional thymidine kinase locus of MVA-BAC. MVA continues to feature prominently in clinical trials of recombinant vaccines against diseases such as HIV-AIDS, malaria and tuberculosis. Here we demonstrate in proof-of-concept experiments that MVA-BAC recombineering is a viable route to more rapid and efficient generation of new candidate mutant and recombinant vaccines based on a clinically deployable viral vector.
\end{abstract}

Citation: Cottingham MG, Andersen RF, Spencer AJ, Saurya S, Furze J, et al (2008) Recombination-Mediated Genetic Engineering of a Bacterial Artificial Chromosome Clone of Modified Vaccinia virus Ankara (MVA). PLoS ONE 3(2): e1638. doi:10.1371/journal.pone.0001638

Editor: Philip G. Stevenson, Cambridge University, United Kingdom

Received January 14, 2008; Accepted January 25, 2008; Published February 20, 2008

Copyright: (c) 2008 Cottingham et al. This is an open-access article distributed under the terms of the Creative Commons Attribution License, which permits unrestricted use, distribution, and reproduction in any medium, provided the original author and source are credited.

Funding: Funded by the Wellcome Trust and a Grand Challenges in Global Health grant from the Foundation for the National Institutes of Health. The funders had no role in study design, data collection and analysis, decision to publish, or preparation of the manuscript.

Competing Interests: The authors have declared that no competing interests exist.

*E-mail: matt.cottingham@well.ox.ac.uk

\section{Introduction}

Modified Vaccinia virus Ankara (MVA) is a replication-deficient attenuated poxvirus that was derived from Vaccinia virus by Mayr et al. through more than 500 blind passages in chick embryo fibroblast (CEF) cell culture [1]. With the notable exception of the Syrian hamster cell line BHK-21 [2], MVA is unable to replicate productively in mammalian cells, though genome replication, late gene expression and immature virion formation usually occur $[3,4]$. During its attenuation, MVA acquired large genomic deletions totalling about $30 \mathrm{~kb}$, resulting in the complete loss of 26 open reading frames (ORFs), together with truncation or fragmentation of a further $21 \mathrm{ORF}$ and numerous smaller scale mutations [5]. MVA does not therefore express many of the known poxviral immune evasion and virulence factors [6-8], though how this determines its abortive phenotype in mammalian cells is not well understood [9]. Despite these features, MVA is at least as immunogenic as conventional replicating Vaccinia virus [10-13] and has the advantage of a considerably improved safety profile [14].
Attention has focussed on MVA not only as a possible 'next generation' smallpox vaccine [15-17], but also as a recombinant vaccine vector, with particular value in combination with other vectors (e.g. DNA, adenovirus, or an avian poxvirus) as a component of heterologous prime-boost regimens designed to elicit high frequencies of antigen-specific $\mathrm{T}$ cells [18]. This approach is undergoing development and clinical trial for diseases such as HIVAIDS [19,20], tuberculosis [21,22] and malaria [23,24], where protective $\mathrm{T}$ cell responses are required for vaccine efficacy. In the case of liver-stage malaria vaccines, specific cytotoxic $\mathrm{T}$ cell responses correlate with the limited levels of protection achieved so far in humans [25], but current vectors and regimens are not immunogenic enough to achieve significant efficacy in malarious regions [26]. There is therefore a need to identify recombinant vaccine vectors with greater immunogenicity, either by using novel vectors (e.g. simian adenoviruses [27]) or by improving existing platforms.

The routes available for attempts to improve MVA vector immunogenicity can be crudely divided into addition or removal of genes. Co-expression of costimulatory molecules, such as B7.1 [28] and 4-1BB ligand [29] and various cytokines, including IL-12 
[30], IL-15 [31] and GM-CSF [32] have been reported to increase recombinant poxvirus vaccine immunogenicity. These interventions can achieve a two- to four-fold increase in peak murine IFN$\gamma \mathrm{T}$ cell responses. The parallel strategy is to delete poxviral genes encoding immunomodulators that attenuate adaptive immune responses, but are not required for growth in vitro. During attenuation MVA lost many such factors, including soluble decoy receptors for IFN- $\gamma$ [33], TNF- $\alpha$ [34], IFN- $\alpha / \beta$ [35] and various chemokines [36]; an inhibitor of IL-1 $\beta$ converting enzyme [37], the complement control protein [38] and an intracellular inhibitor of Toll-like receptor (TLR) and IL-1 receptor (IL-1R) signalling [39]. On the other hand, it retains genes encoding secreted interleukin- [40-42] and chemokine- [36,43] binding proteins; a dehydrogenase involved in steroid synthesis [44,45], a second inhibitor of TLR/IL-1 receptor signalling [46], and other genes implicated in poxviral virulence [47-50]. Evidence that deletion of such genes provides a route for improvement of MVA immunogenicity is provided by reports of augmented protective mouse $\mathrm{CD}^{+} \mathrm{T}$ cell responses elicited by MVA lacking $B 15 R$, encoding an IL-1 $\beta$ binding protein [51], and $A 41 L$, encoding a chemokine binding protein of unknown specificity [43].

In addition to a priori candidates, there are at least 30 MVA genes whose function remains unknown. Although many poxviral proteins retain sequence homology and functional similarity with host proteins, it has recently been shown in the case of Vaccinia virus protein $\mathrm{N} 1$ that preservation of structure and function can occur in the absence of significant sequence similarity [52]. An unbiased experimental approach is therefore required for identification of MVA genes that negatively affect its immunogenicity. In order to expedite this approach, we have constructed a bacterial artificial chromosome (BAC) clone of the MVA genome using the elegant method devised by A. Domi and B. Moss for Vaccinia virus [53], with slight modifications. Recombination-mediated genetic engineering (recombineering) of this construct [54,55] permits more rapid generation of mutants for analysis than can be achieved by traditional methods, with the possible exception of host-range selection on rabbit cells using $K 1 L[56,57]$. To test this concept, we deleted five MVA genes using recombineering of a fully sequenced BAC clone, rescued the constructs to infectious virus, and here demonstrate a modest but significant improvement in immunogenicity of MVA lacking B15R, supporting and extending published work [51]. For large-scale biomanufacturing of an MVA-based vaccine for clinical use, the ability to use clonal purifed BAC DNA as the starting-point, rather than a conventional plaque-purified recombinant, is likely to be of considerable value. With this aim in mind, as well as pre-clinical studies, we demonstrate immunogenicity of a recombinant antigen inserted at the traditional thymidine kinase locus of MVA-BAC by recombineering.

\section{Materials and Methods}

\section{Recombinant MVA construction}

Figure 1 shows the plasmid construct used to generate a recombinant virus (referred to as MVA-BAC-parent) containing the entire sequence of the BAC vector pBELO-BAC11 at the Deletion III locus of MVA (between the remnants of $A 51 R$ and $A 56 R)$ together with a GFP reporter gene driven by the $\mathrm{p} 4 \mathrm{~B}$ late promoter from Fowlpox virus. The fragments required were amplified by PCR with primers designed to introduce restriction sites for assembly by standard methods. The flanking regions for recombination correspond to positions 148412-149083 and 149340-149855 of MVA genome sequence U94848 [5]. The plasmid was linearised with $P a c \mathrm{I}$ and transfected into MVAinfected primary chick embryo fibroblast (CEF) cells (Institute for Animal Health, Compton, UK). The cells were trypsinised and GFP-positive cells were sorted by flow cytometry on a Dako Cytomation MoFlo prior to plaque picking of the recombinant virus to purity, amplification and titration on CEFs. Cells were maintained in Dulbecco's modified Eagle medium (DMEM) supplemented with $10 \%$ FCS or with $2 \%$ FCS for viral growth. Identity and purity were confirmed by PCR. MVA expressing the recombinant antigen TIP was prepared as described (A. Spencer $e t$ al., manuscript in preparation).

\section{Generation of MVA-BAC}

CEF cells in 6-well plates were infected with MVA-BAC-parent at $5 \mathrm{pfu} /$ cell and $2 \mathrm{~h}$ later the inoculum was replaced with growth medium containing $45 \mu \mathrm{M}$ isatin- $\beta$-thiosemicarbazone (I $\beta \mathrm{T})$. The compound was kindly synthesised by Dr J. Robertson (Chemistry Research Laboratory, Oxford University) and was dissolved to $5 \mathrm{mg} / \mathrm{mL}$ in acetone for storage at $-20^{\circ} \mathrm{C}$, then diluted to $1 \mathrm{mg} /$ $\mathrm{mL}$ in $0.25 \mathrm{M} \mathrm{NaOH}$ immediately prior to use. The cells in some wells were transfected with pCI-Cre (obtained from A. Domi, NIAID, NIH, Bethesda, MD) using Lipofectamine (Invitrogen). The DNA was phenol-extracted from the cultures $24 \mathrm{~h}$ later as described [53] and resuspended in $20 \mu \mathrm{l}$ of Tris-EDTA. Of this material, $3 \mu \mathrm{l}$ was electroporated into DH10B E. coli (Invitrogen) prior to selection on LB plates with chloramphenicol $(12.5 \mu \mathrm{g}$ / $\mathrm{mL}$ ) and miniprep by alkaline lysis from liquid LB cultures. Clones were screened by PCR for the presence of the genes MVA005, MVA010, MVA044, MVA086 and MVA188. For restriction mapping and pulsed-field gel electrophoresis, BAC DNA was prepped by QIAgen kit and $0.5-1 \mu \mathrm{g}$ of DNA was digested overnight in a $15 \mu \mathrm{l}$ reaction with the relevant enzymes (NEB). MVA-BAC clone 26 was shotgun sequenced at eightfold coverage by Lark Technologies (Cogenics), Inc.

\section{$B A C$ rescue}

BHK-21 cells (maintained in DMEM $+10 \%$ FCS) were seeded into 6-well plates and infected with FP9-LacZ [58], an attenuated strain of Fowlpox virus expressing $\beta$-galactosidase, at $1 \mathrm{pfu} / \mathrm{cell}$ in Optimem (Gibco). After 1-2 hours, the cells were transfected with $4 \mu \mathrm{g}$ of Qiagen-purified MVA-BAG DNA using Lipofectamine 2000 (Invitrogen) in Optimem. The BAC DNA was stored at $4^{\circ} \mathrm{C}$, was never vortexed, and was pipetted only with cut-off tips. The cells were monitored for GFP expression by epifluorescence microscopy and were harvested by freeze-thaw $4-6$ days later. The

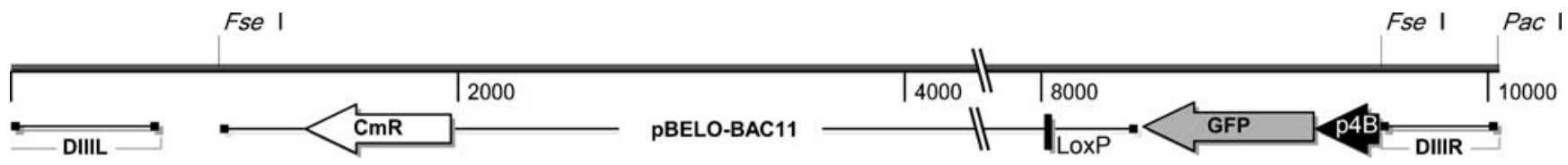

Figure 1. Schematic of construct for recombination into deletion III locus of MVA, based on pBELO-BAC11. DIIIL and DIIIR= left and right flanks for homologous recombination into MVA genome; GFP=green fluorescent protein gene; $p 4 B=$ Fowlpox virus late promoter; $\mathrm{CmR}=$ chloramphenicol acetyltransferase gene for selection in E. coli. Flanking Fsel sites are absent from MVA and permit excision of the cassette. The Pacl site is provided for linearisation of the plasmid prior to recombination with MVA in infected transfected cells. doi:10.1371/journal.pone.0001638.g001 
lysate was used to infect fresh BHK cell monolayers and the cultures were monitored for the appearance of virus growth. Rescued viruses were amplified in BHK cells using DMEM without serum for infection and growth, before purification over sucrose cushions and titration on CEFs.

\section{BAC recombineering}

Galk-based recombineering was done exactly as described by S. Warming et al. [55], from whom the reagents and strains were obtained (see also http://recombineering.ncifcrf.gov/). Sequencing of PCR products and primer synthesis was performed by MWG-Biotech AG.

\section{Mouse immunogenicity}

Female BALB/c and C57BL/6 mice aged 6 to 8 weeks were obtained from the Biomedical Services Unit, Oxford University and procedures were conducted according to the UK Animals (Scientific Procedures) Act 1986. Mice were anaesthetised with ketamine-dormitor prior to intradermal immunisation with $10^{6} \mathrm{pfu}$ of MVA divided into two $25 \mu \mathrm{l}$ injections (one per ear). Splenocytes were harvested as described [58] seven days postimmunisation for flow cytometric analysis or fourteen days postimmunisation for ELIspot analysis. ELIspots were conducted using $18-20 \mathrm{~h}$ stimulation with $1 \mu \mathrm{g} / \mathrm{mL}$ synthetic peptide (ProImmune) in IVPH plates (Millipore) coated with anti-IFN- $\gamma$ antibody AN18 (Mabtech). Spots were developed with R46A2-biotin (Mabtech) followed by streptavidin alkaline phosphatase (Mabtech) and substrate kit (BioRad) and enumerated on an automated reader (Autoimmun Diagnostika). For flow cytometry, cells were stimulated for $6 \mathrm{~h}$ with $1 \mu \mathrm{g} / \mathrm{mL}$ peptide in the presence of $2 \mu \mathrm{l} /$ $\mathrm{mL}$ Golgi-Plug (BD) and stained with anti-CD8 pacific blue conjugate and anti-CD4 APC-Alexa-750 conjugate prior to fixation in $10 \%$ neutral buffered formalin (Sigma). Intracellular cytokine staining was performed using FITC-conjugated anti TNF- $\alpha$, PE-conjugated anti-IL-2 and Alexa-647-conjugated antiIFN- $\gamma$ diluted in Cytoperm (BD). All antibodies were obtained from eBiosciences. Data were acquired on a CyAn flow cytometer (Dako) and analysed using FlowJo (Treestar). PESTLE and SPICE software were obtained from Dr M. Roederer, Vaccine Research Centre, NIH. Other statistical analyses were performed using Prism (GraphPad Software, Inc.).

\section{Results}

\section{Cloning of MVA genome into BAC}

A procedure very similar to that developed by Domi and Moss for VAC-BAC [53] was used to create BACs containing the genome of MVA. First, a cassette containing pBELO-BAC11, a LoxP recombinase site and a GFP reporter gene driven by a late poxviral promoter (see Figure 1) was inserted into the deletion III locus of MVA by conventional recombination in infected CEF cells. Cells infected with this recombinant virus, referred to at MVA-BACparent, were treated with isatin- $\beta$-thiosemicarbazone $(\mathrm{I} \beta \mathrm{T})$ to inhibit viral hairpin resolution and promote genome concatemerisation [59]. After transformation of E. coli with DNA from these cells, dozens of chloramphenicol-resistant colonies were observed regardless of treatment of infected cells with $\mathrm{I} \beta \mathrm{T}$. Unexpectedly, transfection of cells with pCI-Cre prior to infection in an attempt to induce recombination between LoxP sites to produce circular molecules from head-to-tail concatemers [53] dramatically decreased the number of colonies obtained, probably due to cytotoxicity of the transfection reagent. Alternatively, it is possible that cryptic LoxP sites such as those present in mammalian genomes [60] resulted in illegitimate recombination events.
Colonies were screened for potential full-length clones by PCR at five loci spaced along the genome, and candidates were further characterised by restriction mapping with HindIII and XhoI and pulsed-field gel electrophoresis after digestion with FseI to excise the BAC cassette (data not shown). No full-length clones were isolated from pCI-Cre transfected samples (of 5 colonies screened), but from untransfected cells, four apparently full-length clones were obtained, three derived from I $\beta$ T-treated cells (of 24 colonies screened) and one from untreated cells (of 18 colonies screened). This frequency is very similar to that observed for VAC-BAC [53].

\section{$B A C$ rescue}

The next step was to "rescue" the BAC clones to infectious MVA using a Fowlpox virus helper to provide transcriptional machinery. The BHK cell line, which is the only mammalian cell line known to support MVA replication, is ideal for this purpose since it is nonpermissive for the host-range restricted avipoxviruses. Following transfection into BHK cells infected with Fowlpox virus, all four of the MVA-BAC clones were converted to infectious MVA able to replicate and form plaques on BHK and CEF cells. We have not conducted formal growth rate analysis of the resulting viruses, but viral titres of $8.0 \times 10^{8}$ to $2.4 \times 10^{9} \mathrm{pfu} / \mathrm{mL}$ (final volume $\sim 0.5 \mathrm{~mL}$ ) were achieved following sucrose cushion purification from $\mathrm{BHK}$ cultures totalling $1500 \mathrm{~cm}^{2}$. The titrations were performed on GEF cells, in which Fowlpox virus can replicate, and absence of significant residual Fowlpox virus was demonstrated by X-Gal staining for the LacZ marker gene present in the helper virus.

\section{Immunogenicity of MVA-BACs}

The principal utility of MVA is as a vaccine vector, particularly for eliciting $\mathrm{T}$ cell responses. The immunogenicity of the viruses derived from the four MVA-BAC clones was therefore assessed in mice by determining $\mathrm{CD}^{+} \mathrm{T}$ cell responses to viral antigens following intradermal inoculation. Tscharke et al. have described in detail the immunodominance hierarchy of $\mathrm{CD}^{+} \mathrm{T}$ cell "determinants" (i.e., epitopes) following infection of BALB/c and C57BL/6 mice with Vaccinia virus, MVA and other poxviruses $[61,62]$. We used the described synthetic peptides to stimulate splenocytes from immunised BALB/c or C57BL/6 mice and quantified antigen-specific $\mathrm{T}$ cell responses by IFN- $\gamma$ ELIspot assay (Figure 2).

Mice were immunised intradermally with the four MVA-BAC clones, the MVA-BAC-parent (from which the BAC clones were derived), or MVA (i.e. virus lacking the BAC cassette). In C57BL/ 6 mice, all were immunogenic and there was no statistically significant difference by one-way ANoVA between the $\mathrm{T}$ cell responses to any of these viruses for each of the five available determinants. In BALB/c mice, the same was true for the immunodominant determinant E3, but there were significant differences in the subdominant determinant specific $\mathrm{F} 2(\mathrm{G})$ responses $(\mathrm{p}<0.01$, one-way ANoVA), and a post-hoc test revealed that the statistically significant pairwise comparisons are clone \#26 versus parent or MVA ( $<<0.05$, Newman-Keuls multiple comparison test). Despite the lack of conventional significance for other clones, there is a trend towards higher responses to BACderived MVA in BALB/c mice, but this is not an effect of the presence of the BAC cassette at deletion III, since MVA-BACparent also contains this insertion.

The inability to distinguish the MVA-BAC clones by restriction map, viral growth yield or murine immunogenicity suggests that they are likely to be identical, but the possibility of some bias in the isolation procedure necessitates verification of clone fidelity by complete sequencing, especially prior to embarking on a programme of genetic manipulation. 
C57BL/6

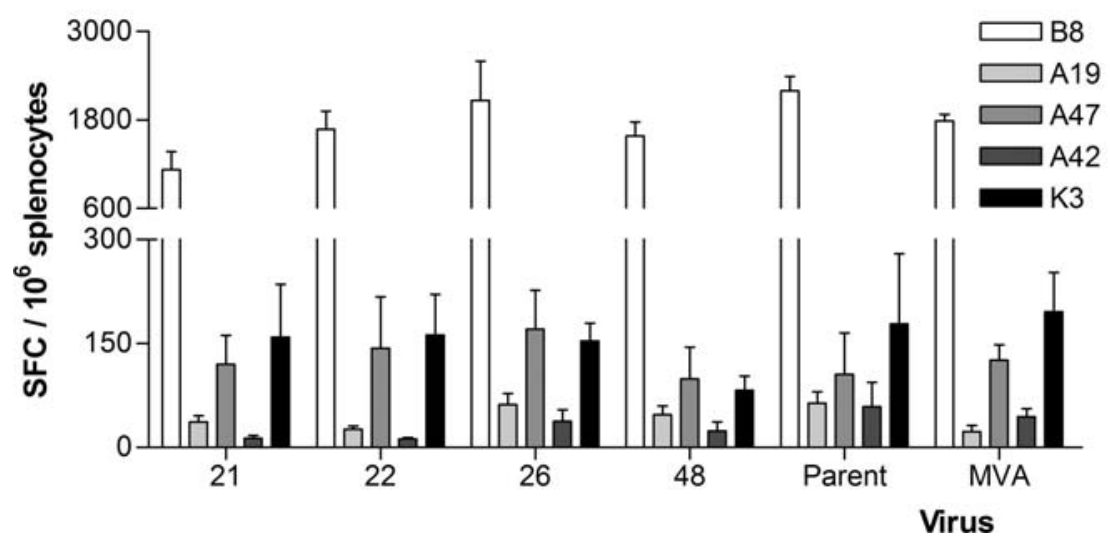

Balb/c

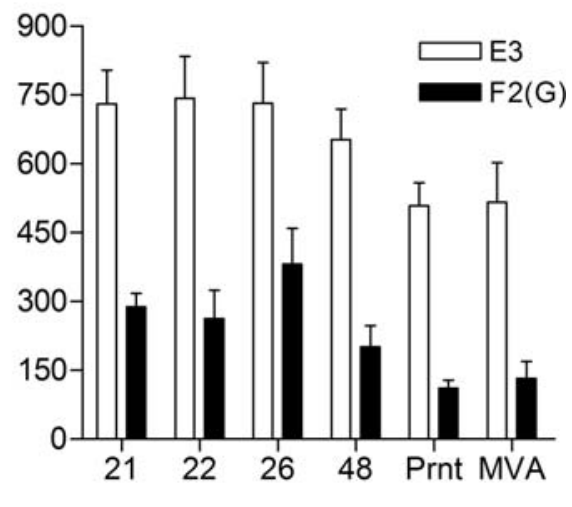

Figure 2. Immunogenicity of four MVA-BAC clones $(21,22,26,48)$ in C57BL/6 and BALB/c mice compared to parent virus (containing the BAC cassette) and MVA (lacking the BAC cassette). Mice were immunised with $10^{6}$ pfu i.d. and the specific T cell responses to the indicated endogenous synthetic peptide epitopes [61,62] were measured 14 days later by IFN- $\gamma$ ELIspot assay. Data are means and SEM from groups of four mice.

doi:10.1371/journal.pone.0001638.g002

\section{Sequence of MVA-BAC}

Three MVA genome sequences are available in GenBank, one published by Antoine et al. [5] (U94848), one deposited by Bavarian Nordic GmbH (DQ983236) and the "Acambis 3000" strain (AY603355) deposited by the CDC. The Bavarian Nordic sequence is identical to the Acambis sequence, and these differ from Antoine et al.'s sequence only by five single nucleotide substitutions (excluding the repeat regions of the inverted terminal repeats, which are not fully described in the Acambis and Bavarian Nordic sequences). All of these loci are very polymorphic amongst strains of Vaccinia virus (http://www.poxvirus.org/). The mutations and genes affected are shown in Table 1.

MVA-BAC clone \#26 was selected for shotgun sequencing at eightfold coverage, resulting in a single contig (excluding the repeat regions), and was found to be identical to the Acambis/ Bavarian Nordic strain, with the exception of a 6 bp insertion in the non-essential gene $F 7 L$ [63]. The mutation codes for an extra Asn-Lys repeat and makes MVA-BAG identical to the TanTien strain of Vaccinia virus at this polymorphic locus, where other strains have two Asn-Lys repeats, with the exception of the Copenhagen strain which has eight (http://www.poxvirus.org/). In order to determine at what point in the genesis of MVA-BAC this mutation occurred, we sequenced the progenitor viruses at this locus by PCR amplification from genomic DNA. MVA-BAC- parent was found to possess the $6 \mathrm{bp}$ insertion mutation, but its progenitor (from which the recombinant was derived) did not. Sequencing of PCR products revealed that a selection of other MVA recombinants from our laboratory and our original stock provided by Anton Mayr did not possess the mutation. It is not clear whether this $6 \mathrm{bp}$ insertion arose during production of the MVA-BAC parent, or represents isolation of a rare genotype present at low levels in the source MVA. Evidence for genetic heterogeneity within poxvirus strains $[64,65]$ (personal communication, R. Regnery) favours the latter possibility.

\section{Deletion of MVA genes by BAC recombineering}

Recombination mediated genetic engineering (recombineering) permits modification of BAC DNA by homologous recombination in E. coli. We used the system described by Warming et al. [55], which is based on stringent temperature-sensitive expression of the $\lambda$ Red genes exo, bet and gam coupled with GalK-based positive and negative selection. A similar approach, not using $\mathrm{GalK}$, has been described for VAC-BAC by Domi and Moss [54], who also verified the stability of their Vaccinia virus BAC clone after induction of the $\lambda$ Red system. Five genes reported to affect MVA immunogenicity or Vaccinia virus immunogenicity or virulence were selected from the literature for deletion by insertion of GalK in place of the ORF (see Table 2). Long oligonucleotide primers

Table 1. Polymorphisms in MVA and MVA-BAC clone \#26.

\begin{tabular}{|c|c|c|c|c|c|}
\hline Position $^{a}$ & Gene affected & Mutation & Coding change & MVA strains possessing mutation ${ }^{b}$ & $\begin{array}{l}\text { Example Vaccinia virus strain } \\
\text { possessing mutation }^{c}\end{array}$ \\
\hline 68740 & G6R & $A>G$ & $N>D$ & A/BN, MVA-BAC & Lister, WR, TAN \\
\hline 73886 & $L 3 L$ & $C>T$ & $\mathrm{R}>\mathrm{K}$ & $\mathrm{A} / \mathrm{BN}, \mathrm{MVA}-\mathrm{BAC}$ & WR \\
\hline 108489 & $A 4 L$ & $\mathrm{C}>\mathrm{T}$ & Silent & A/BN, MVA-BAC & WR \\
\hline 114308 & $A 10 L$ & $\mathrm{~T}>\mathrm{C}$ & Silent & A/BN, MVA-BAC & WR \\
\hline 114945 & $A 10 L$ & $\mathrm{C}>\mathrm{T}$ & $\mathrm{R}>\mathrm{K}$ & A/BN, MVA-BAC & Lister \\
\hline 30326 & $F 7 L$ & 6 bp ins. & NK ins. & MVA-BAC & TAN \\
\hline
\end{tabular}

${ }^{\mathrm{a}}$ Refers to residue numbering of MVA genome sequence U94848 [5].

${ }^{\mathrm{b}} \mathrm{A} / \mathrm{BN}=$ Acambis/Bavarian Nordic (see text).

${ }^{c} \mathrm{WR}=$ Western Reserve; $\mathrm{COP}=$ Copenhagen; $\mathrm{TAN}=$ TanTien.

doi:10.1371/journal.pone.0001638.t001 
Table 2. MVA-BAC genes deleted by GalK recombineering.

\begin{tabular}{|c|c|c|c|c|c|}
\hline Gene & Function of encoded protein & Refs & Left homology arm (bp) ${ }^{a}$ & Right homology arm (bp) ${ }^{a}$ & Mutations in MVA $^{\mathbf{b}}$ \\
\hline C12L & IL-18 binding protein & {$[41,42]$} & 13195-13224 & 13415-13464 & $6 \mathrm{bp}$ in-frame deletion \\
\hline$A 44 L$ & Hydroxysteroid dehydrogenase & {$[44,45]$} & $142608-142657$ & 143549-143598 & 1 amino acid substitution \\
\hline A46R & Inhibitor of Toll-like receptor signalling & [46] & 143941-143990 & 144699-144748 & - \\
\hline$B 7 R$ & Chemokine binding protein $\left(\mathrm{SCP}-3^{\mathrm{C}}\right)$ & [36] & 156989-157038 & 157564-157613 & $15 \mathrm{bp}$ in-frame deletion \\
\hline B15R & IL-1 $\beta$ binding protein & [51] & 161971-162020 & 162999-163048 & - \\
\hline
\end{tabular}

${ }^{\mathrm{a}}$ Refers to residue numbering of MVA genome sequence U94848 [5].

${ }^{b}$ Coding mutations specific to MVA, i.e. not known in any other strain of Vaccinia virus.

'Smallpox virus encoded chemokine receptor (SECRET) domain containing protein 3.

doi:10.1371/journal.pone.0001638.t002

were used to introduce 50 bp homology arms at the $5^{\prime}$ and $3^{\prime}$ ends of GalK by PCR and these products were electroporated into heatinduced SW102 cells carrying MVA-BAC prior to selection on galactose minimal medium, as described [55]. Before rescue by transfection into Fowlpox virus infected BHK cells, as above, the modified BACs were checked by restriction digest with HindIII and $X h o \mathrm{I}$ and by sequencing two PCR products spanning the junctions at the GalK insertion. In only one case (the $A 46 R$ deletion), was any mutation detected: a primer-derived single nucleotide deletion in the intergenic region downstream of the insertion. This mutation was ignored as it did not fall within any predicted regulatory sequences (promoters or terminators). GalK deletion mutant BACs were also checked for absence of undeleted contaminants by PCR across the deleted locus. All five modified GalK-carrying MVA-BACs converted into infectious virus, and again, though we did not formally compare growth rates, all were amplified to titres of $2.8 \times 10^{8}$ to $2.6 \times 10^{9} \mathrm{pfu} /$ $\mathrm{mL}$ (final volume $\sim 0.5 \mathrm{~mL}$ ) following sucrose cushion purification from $1500 \mathrm{~cm}^{2}$ of BHKs.

\section{Immunogenicity of MVA-BAC deletion mutants}

The effect of the above modifications on immunogenicity was assessed in intradermally immunised BALB/c mice. Peak specific $\mathrm{T}$ cell responses were analysed seven days later by flow cytometry with intracellular cytokine staining following stimulation with the F2(G) and E3 peptides (Figure 3). No statistically significant

d7

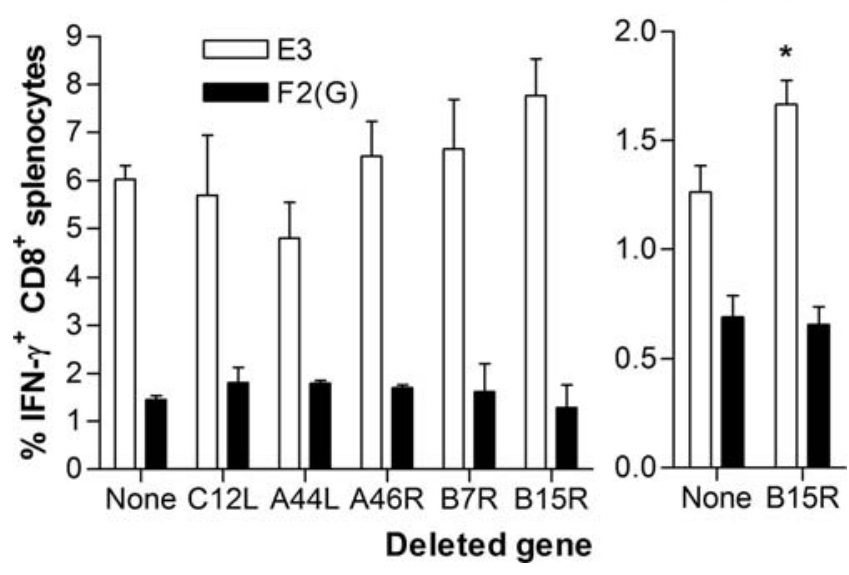

Figure 3. Peptide-specific splenic $\mathrm{CDB}^{+} \mathrm{T}$ cell responses 7 days (d7) or 56 days (d56) after i.d. immunisation of BALB/c mice with $10^{6} \mathrm{pfu}$ of MVA-BAC derived viruses, either unmodified or with indicated genes deleted by insertion of GalK. * $p=0.039$ $(t$-test). Data are means and SEM from groups of four mice. doi:10.1371/journal.pone.0001638.g003 differences between groups were observed (one-way ANoVA for each epitope). Of the selected genes, only $B 15 R$, which encodes an IL-1 $\beta$-binding protein, has been reported to affect MVA immunogenicity, rather than Vaccinia virus immunogenicity or virulence [51]. At the peak of the response, Staib et al. described a small nonsignificant effect of $B 15 R$ deletion, very similar to that shown in Figure 3 for the E3 epitope, and with an identical p-value by $t$-test $(p=0.07)$; however, in the memory phase six months postvaccination, they observed a highly significant $\sim 4$.5-fold increase in $\mathrm{CD}^{+} \mathrm{T}$ cell response to the $B 15 R$ deletion mutant. We therefore assessed memory responses at 8 weeks post-vaccination, and observed a small, but statistically significant increase in $\mathrm{CD}^{+} \mathrm{T}$ cell responses to E3 ( $p=0.039, t$-test), but not to F2(G). As well as the readout time, variation in dose $\left(10^{8}\right.$ vs. $\left.10^{6} \mathrm{pfu}\right)$, route (i.p. vs. i.d.), antigen (H3 vs. E3 peptide) and mouse strain (HHD vs. BALB/c) are likely to underlie the suggested difference in the magnitude of the effect of $B 15 R$ deletion on memory $\mathrm{T}$ cell responses.

In order to characterise the effect of $B 15 R$ deletion further, we analysed E3-specific CD8 ${ }^{+} \mathrm{T}$ cell expression of TNF- $\alpha$ and IL-2 in addition to IFN- $\gamma$. SPICE software [66] was used to discriminate individual cells expressing the seven different 'Boolean' combinations of cytokines from the IFN- $\gamma^{+}, \mathrm{TNF}-\alpha^{+}$and $\mathrm{IL}-2^{+}$gated populations (Figure 4). This approach reveals that at the peak of the response (d7), most of the cells are IFN- $\gamma$ and TNF- $\alpha$ doublepositives. Furthermore, although there is no conventionally significant difference, the increase in bulk IFN- $\gamma$ responses observed with the $B 15 R$ deletion mutant is primarily the result of an increased number of these double-positive cells, rather than of the IFN- $\gamma$ single-positive or the IFN- $\gamma$, TNF- $\alpha$ and IL-2 triplepositive cells that account for the remainder of the $\mathrm{CD}^{+} \mathrm{T}$ cell response. By contrast, at day 56 , the effect of $B 15 R$ deletion is seen to reside mainly within the triple-positive population, and the difference is statistically significant $(p=0.018$, Willcoxon-Rank test). Deletion of $B 15 R$ therefore affects both the quality and magnitude of the E3-specific $\mathrm{CD}^{+} \mathrm{T}$ cell response to MVA. Enhanced production of the mitogenic cytokine IL-2 during the early memory phase (8 weeks) may underpin the large difference in $\mathrm{T}$ cell frequency observed in Staib et al.'s 6 month experiment [51].

\section{Insertion of a recombinant antigen into MVA by BAC recombineering}

In addition to more rapid production of deletion mutants, the MVA-BAC system would also have value as a faster method for insertion of genes encoding exogenous protective antigens or candidate 'molecular adjuvants' with the potential to increase vector immunogenicity. The elegance of the GalK based recombineering system [55] relies on the ability to counterselect for clones lacking Galk using deoxygalactose, which is metabolised to a toxic 
d7

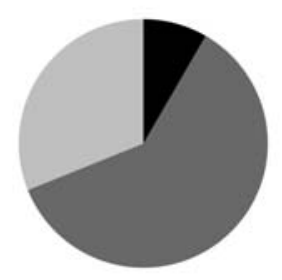

Unmodified

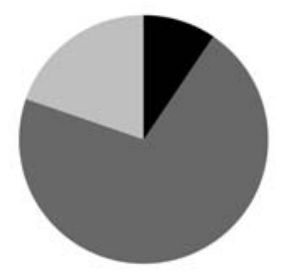

$B 15 R$ deletion

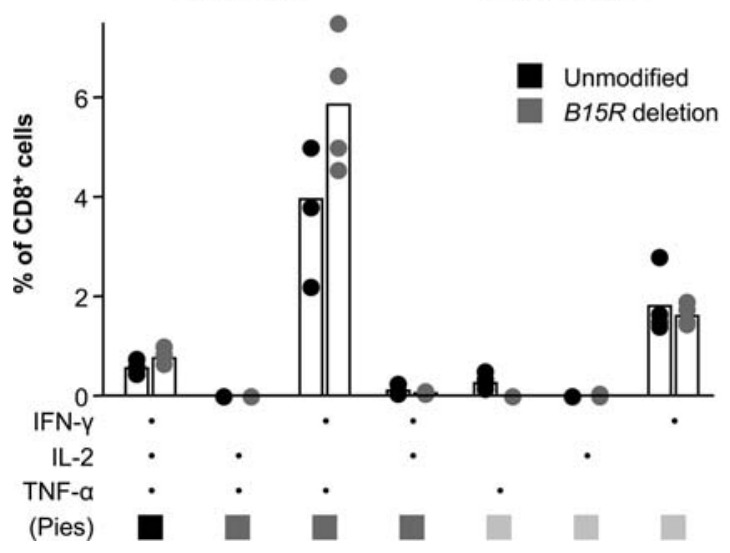

d56
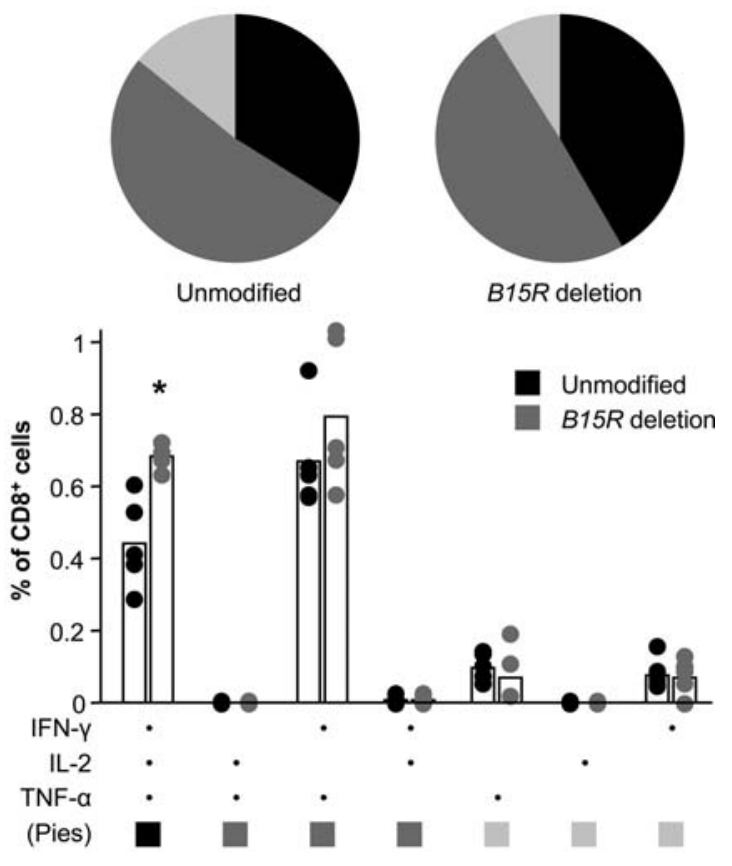

Figure 4. Multifunctionality of $\mathrm{CDB}^{+} \mathrm{T}$ cells induced 7 days (d7) or 56 days (d56) after immunisation of BALB/c mice with unmodified MVA-BAC or B15R deletion mutant. Histograms show frequency of cells expressing each of the seven possible combinations of IFN$\gamma, \mathrm{IL}-2$ and TNF- $\alpha$ with bars showing the mean and circles showing the values for individual mice $(\mathrm{n}=4)$. Pie charts show fraction of the total response comprised of cells expressing all three cytokines (black), any two cytokines (dark gray), or one cytokine only (light gray). ${ }^{*} p=0.018$, Willcoxon-Rank test. Analysis performed using SPICE software [66].

doi:10.1371/journal.pone.0001638.g004

intermediate. This enables insertion of genes without the need for a selectable marker. However, loss of GalK can occur both by recombination with an electroporated DNA, or by deletion of a segment of the BAC. Unlike GalK insertion, which has an efficiency close to $100 \%$ in MVA-BAC, the specific replacement of $G a l K$ with an exogenous sequence has been achieved to date at a frequency of only up to $1 \%$ compared to spontaneous deletions.

We used this technique to insert an antigen expression cassette carrying no tandem reporter gene or selectable marker into the traditional thymidine kinase (TK) locus of MVA-BAC. This construct comprised the p7.5 early/late promoter driving an 82 amino acid epitope string, named "TIP" (for Tuberculosis, Immunodeficiency virus and Plasmodium), which contains relevant epitopes from various pathogens for vaccine-induced protection in mouse models, including the protective $\mathrm{H}-2 \mathrm{~K}^{\mathrm{d}}$ restricted $\mathrm{Pb} 9$ epitope from Plasmodium berghei circumsporozoite protein [67]. Figure 5 shows that $\mathrm{Pb} 9$-specific $\mathrm{CD}^{+} \mathrm{T}$ cell responses are elicited in MVA-BAC-TIP immunised mice to a level comparable with those elicited by conventional MVA-TIP. Although not statistically significant, there is a trend toward higher $\mathrm{Pb} 9$ responses $(\mathrm{p}=0.07, t$-test $)$ and lower $\mathrm{E} 3$ responses $(\mathrm{p}=0.11, t$ test) in the conventionally derived MVA-TIP compared to the BAC-derived virus. This difference in immunodominance hierarchy may be attributable to differences in the nature of the cassette inserted at the TK locus (the lack of a late-promoter-driven GFP marker gene and opposite orientation of the TIP gene) or to the presence of the BAC cassette at deletion III.

\section{Discussion}

Traditional methods for genetic manipulation of poxviruses, either for mutagenesis or expression of exogenous genes, rely on onerous plaque purification of rare recombinant viruses. Building on the results of Domi and Moss for VAC-BAC [53,54], here we circumvent this requirement by creating an MVA-BAC that can be manipulated using recombineering and rescued to clonal

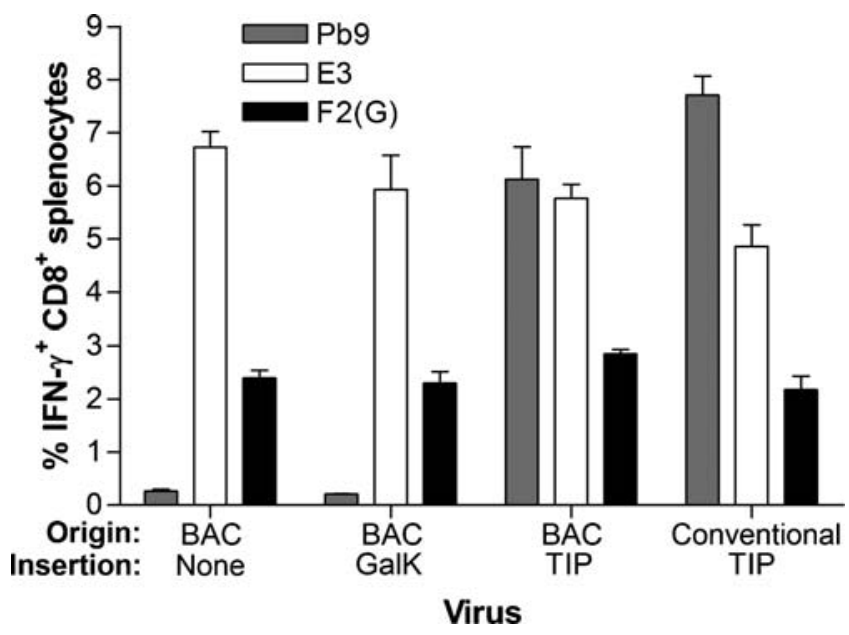

Figure 5. Immunogenicity of MVA-BAC expressing a recombinant antigen inserted at the standard thymidine kinase insertion site, in comparison to conventional recombinant MVA and recombineering precursors containing no insertion or GalK. The recombinant antigen, TIP, is an epitope string containing the $\mathrm{Pb} 9$ epitope from Plasmodium berghei circumsporozoite protein [67]. Bars show mean specific $\mathrm{CD}^{+}{ }^{\mathrm{T}}$ cell responses to the indicated peptides 7 days after i.d. vaccination of with $10^{6} \mathrm{pfu}$, from groups of four BALB/c mice, with error bars showing SEM. doi:10.1371/journal.pone.0001638.g005 
viruses. With an efficiency similar to that observed in the case of VAC-BAC, we generated four MVA-BAC clones, which were identical by restriction map and had indistinguishable $\mathrm{CD}^{+} \mathrm{T}$ cell immunogenicity in two strains of mice. We sequenced one clone, and found it to be identical to published genomic sequences of MVA, with the exception of a minor mutation that is polymorphic amongst Vaccinia virus strains and was present in the parent MVA recombinant. We did not conduct an investigation into stability of the construct, since good stability had been shown for the even larger VAC-BAC construct $[53,54]$.

Of five candidate genes selected from the literature for deletion by MVA-BAC recombineering (Table 2), only one had any statistically significant effect on responses to viral $\mathrm{CD} 8^{+} \mathrm{T}$ cell epitopes following intradermal immunisation of BALB/c mice. The effect of $B 15 R$ deletion was more pronounced 8 weeks post-vaccination, though not as great as was previously reported at a 6 month timepoint in HHD mice [51]. Although we did not observe an effect of similar magnitude using an earlier readout, we show using SPICE polychromatic flow cytometry analysis that antigen-specific $\mathrm{CD}^{+}$ T cells from mice immunised with MVA lacking B15R are composed of a higher proportion of triple-positive cells that express IL-2 in addition to IFN- $\gamma$ and TNF- $\alpha$. This 'multi-functional' phenotype may be responsible for the large differences in cell frequency at 6 months post-vaccination [51], since IL-2 is strongly implicated in memory $\mathrm{T}$ cell homeostasis [68] and its expression by virus-specific $\mathrm{CD}^{+} \mathrm{T}$ cells has been reported to promote antigen-specific proliferation of these cells even in the absence of $\mathrm{CD} 4^{+} \mathrm{T}$ cell help [69]. Whether responses to a recombinant antigen will be augmented in a $B 15 R$ deletion background remains to be tested, though it should be noted in this regard that enhancement of antivector responses was confined to the E3-specific response and was not observed in the case of the F2(G) epitope.

Deletion of four other genes by GalK insertion in MVA-BAC did not potentiate $\mathrm{CD}^{+} \mathrm{T}$ cell responses to the vector epitopes. $C 12 \mathrm{~L}$, $A 44 R$ and $B 7 R$ all carry MVA-specific mutations that do not occur in any other sequenced strain of Vaccinia virus. Although strain Ankara (from which MVA was derived) has not been sequenced, these mutations presumably arose during attenuation, and the genes affected may therefore already be inactive. Alternatively, or additionally, functional redundancy of families of immunomodulatory genes acting on the same pathways may negate the effect of the deletion mutants. Indeed, poxviruses appear to have taken a grapeshot-like approach to disruption of the TLR/IL-1R signalling pathway [6], though MVA's gun has been spiked by deletion of many components, including $A 52 R$, which like $A 46 R$ can inhibit NF$\kappa \mathrm{B}$ activation, albeit by a distinct subset of stimuli [46].

The deletion of these genes also did not impair the $\mathrm{CD}^{+} \mathrm{T}$ cell responses to the vector, suggesting that, as well as being dispensable for growth in BHK cell culture, they are not required for circumvention of host defence mechanisms operating in vivo (though since MVA is non-replicating in mammals, this conclusion does not extend to poxviral pathogenesis). If multiple MVA genes were to be deleted, in order to overcome putative functional redundancy, one might assume that at some point the virus would be so crippled as to be only very poorly immunogenic, for example by an antiviral response occurring early during the infection cycle, which normally proceeds to immature virion formation even in non-permissive cells. On the other hand, the major evolutionary driving force for

\section{References}

1. Mayr A, Stickl H, Muller HK, Danner K, Singer H (1978) [The smallpox vaccination strain MVA: marker, genetic structure, experience gained with the parenteral vaccination and behavior in organisms with a debilitated defence mechanism (author's transl)]. Zentralbl Bakteriol [B] 167: 375-390. acquisition of immunomodulators by poxviruses is more likely productive replication of a virulent virus, rather than evasion of adaptive responses (i.e. antibody or cytotoxic $\mathrm{T}$ cell responses). The MVA-BAC recombineering system will hopefully be versatile and rapid enough to allow these subtleties to be addressed, especially as it is likely to speed up the production of 'revertant' viruses in order to confirm the genetic specificity of a novel phenotype. Furthermore, the ability to make multiple modifications by 'recycling' the GalK dual-selectable marker is likely to be of particular value.

Other applications of MVA-BAC include production of viruses carrying recombinant antigens and/or molecular adjuvants for pre-clinical evaluation and clinical trial. The use of BAC DNA and an inactivated clinical grade helper virus as the input to a manufacturing process could reduce the burden of traceability currently required by regulatory authorities, though a method for efficient removal of the BAC cassette would be needed (see Figure 1). In this setting, the issues of construct stability and helper virus contamination would doubtless require more rigorous investigation than described here. Insertion of antigen expression cassettes by recombineering additionally circumvents the requirement for a reporter gene or selectable marker and its removal by transient-dominant selection [70] when a markerless product is required. In this regard, the difficulty of insertion of the TIP antigen by GalK counterselection is rather disappointing, though there is very likely room for improvement, for example by using longer homology arms in the targeting DNA, or electroporating maximal quantities of DNA. When removing GalK without concomitant insertion of DNA (i.e., from a deletion locus), we have achieved much higher recombineering efficiencies of up to $95 \%$ (data not shown). Alternatively, recombination in $\operatorname{RecA}^{+} E$. coli [71] is a potential means of inserting elements without the use of a selectable marker, since several rare restriction sites (required for linearisation of the BAC prior to recombination) are absent from the MVA genome. For pre-clinical work, an in vitro site specific recombination system could be developed [72].

The vaccine vector capability of Vaccinia virus expressing a recombinant antigen was demonstrated nearly 25 years ago $[73,74]$. Successful vectored vaccines for diseases such as malaria, HIV-AIDS and tuberculosis will likely require combinations of antigens and delivery systems able to induce immune responses of the right character and magnitude against the recombinant antigens. Currently, MVA is one of the more promising deployable delivery systems and has potential for improvement. The MVA-BAC technology provides an opportunity to accelerate production of candidate vaccines for evaluation, and potentially a superior starting point for clinical vaccine manufacture.

\section{Acknowledgments}

We thank Arban Domi and Bernard Moss for plasmids; Richard WadeMartins and Michele Lufino for advice and help, Jeremy Robertson for synthesis of $\mathrm{I} \beta \mathrm{T}$ and Søren Warming for recombineering materials and advice.

\section{Author Contributions}

Conceived and designed the experiments: SG MC AS. Performed the experiments: MC JF SS AS RA. Analyzed the data: MC AS. Wrote the paper: MC. Other: Principal Investigator: AH.
2. Drexler I, Heller K, Wahren B, Erfle V, Sutter G (1998) Highly attenuated modified vaccinia virus Ankara replicates in baby hamster kidney cells, a potential host for virus propagation, but not in various human transformed and primary cells. J Gen Virol 79 (Pt 2): 347-352. 
3. Carroll MW, Moss B (1997) Host range and cytopathogenicity of the highly attenuated MVA strain of vaccinia virus: propagation and generation of recombinant viruses in a nonhuman mammalian cell line. Virology 238: 198-211.

4. Okeke MI, Nilssen O, Traavik T (2006) Modified vaccinia virus Ankara multiplies in rat IEC-6 cells and limited production of mature virions occurs in other mammalian cell lines. J Gen Virol 87: 21-27.

5. Antoine G, Scheiflinger F, Dorner F, Falkner FG (1998) The complete genomic sequence of the modified vaccinia Ankara strain: comparison with other orthopoxviruses. Virology 244: 365-396.

6. Haga IR, Bowie AG (2005) Evasion of innate immunity by vaccinia virus. Parasitology 130 Suppl: S11-25.

7. Seet BT, Johnston JB, Brunetti CR, Barrett JW, Everett H, et al. (2003) Poxviruses and immune evasion. Annu Rev Immunol 21: 377-423.

8. Webb LM, Alcami A (2005) Virally encoded chemokine binding proteins. Mini Rev Med Chem 5: 833-848.

9. Wyatt LS, Carroll MW, Czerny CP, Merchlinsky M, Sisler JR, et al. (1998) Marker rescue of the host range restriction defects of modified vaccinia virus Ankara. Virology 251: 334-342.

10. Sutter G, Wyatt LS, Foley PL, Bennink JR, Moss B (1994) A recombinant vector derived from the host range-restricted and highly attenuated MVA strain of vaccinia virus stimulates protective immunity in mice to influenza virus. Vaccine 12: $1032-1040$.

11. Gomez CE, Rodriguez D, Rodriguez JR, Abaitua F, Duarte C, et al. (2001) Enhanced CD8+ T cell immune response against a V3 loop multi-epitope polypeptide (TAB13) of HIV-1 Env after priming with purified fusion protein and booster with modified vaccinia virus Ankara (MVA-TAB) recombinant: a comparison of humoral and cellular immune responses with the vaccinia virus Western Reserve (WR) vector. Vaccine 20: 961-971.

12. Hirsch VM, Fuerst TR, Sutter G, Carroll MW, Yang LC, et al. (1996) Patterns of viral replication correlate with outcome in simian immunodeficiency virus (SIV)-infected macaques: effect of prior immunization with a trivalent SIV vaccine in modified vaccinia virus Ankara. J Virol 70: 3741-3752.

13. Ramirez JC, Gherardi MM, Esteban M (2000) Biology of attenuated modified vaccinia virus Ankara recombinant vector in mice: virus fate and activation of Band T-cell immune responses in comparison with the Western Reserve strain and advantages as a vaccine. J Virol 74: 923-933.

14. Drexler I, Staib C, Sutter G (2004) Modified vaccinia virus Ankara as antigen delivery system: how can we best use its potential? Curr Opin Biotechnol 15: $506-512$.

15. Earl PL, Americo JL, Wyatt LS, Eller LA, Whitbeck JC, et al. (2004) Immunogenicity of a highly attenuated MVA smallpox vaccine and protection against monkeypox. Nature 428: 182-185.

16. Mayr A (2003) Smallpox vaccination and bioterrorism with pox viruses. Comp Immunol Microbiol Infect Dis 26: 423-430.

17. Cosma A, Nagaraj R, Staib C, Diemer C, Wopfner F, et al. (2007) Evaluation of modified vaccinia virus Ankara as an alternative vaccine against smallpox in chronically HIV type 1-infected individuals undergoing HAART. AIDS Res Hum Retroviruses 23: 782-793.

18. Gilbert SC, Moorthy VS, Andrews L, Pathan AA, McConkey SJ, et al. (2006) Synergistic DNA-MVA prime-boost vaccination regimes for malaria and tuberculosis. Vaccine 24: 4554-4561.

19. Hanke T, McMichael AJ, Dorrell L (2007) Clinical experience with plasmid DNA- and modified vaccinia virus Ankara-vectored human immunodeficiency virus type 1 clade A vaccine focusing on T-cell induction. J Gen Virol 88: 1-12.

20. Kent S, De Rose R, Rollman E (2007) Drug evaluation: DNA/MVA primeboost HIV vaccine. Curr Opin Investig Drugs 8: 159-167.

21. McShane H, Hill A (2005) Prime-boost immunisation strategies for tuberculosis. Microbes Infect 7: 962-967.

22. Skeiky YA, Sadoff JC (2006) Advances in tuberculosis vaccine strategies. Nat Rev Microbiol 4: 469-476.

23. Hill AV (2006) Pre-erythrocytic malaria vaccines: towards greater efficacy. Nat Rev Immunol 6: 21-32.

24. Reyes-Sandoval A, Harty JT, Todryk SM (2007) Viral vector vaccines make memory $\mathrm{T}$ cells against malaria. Immunology 121: 158-165.

25. Keating SM, Bejon P, Berthoud T, Vuola JM, Todryk S, et al. (2005) Durable human memory $\mathrm{T}$ cells quantifiable by cultured enzyme-linked immunospot assays are induced by heterologous prime boost immunization and correlate with protection against malaria. J Immunol 175: 5675-5680.

26. Bejon P, Mwacharo J, Kai O, Mwangi T, Milligan P, et al. (2006) A phase 2b randomised trial of the candidate malaria vaccines FP9 ME-TRAP and MVA ME-TRAP among children in Kenya. PLoS Clin Trials 1: e29.

27. Reyes-Sandoval A, Fitzgerald JC, Grant R, Roy S, Xiang ZQ et al. (2004) Human immunodeficiency virus type 1 -specific immune responses in primates upon sequential immunization with adenoviral vaccine carriers of human and simian serotypes. J Virol 78: 7392-7399.

28. Garnett CT, Greiner JW, Tsang KY, Kudo-Saito C, Grosenbach DW, et al. (2006) TRICOM vector based cancer vaccines. Curr Pharm Des 12: 351-361.

29. Kudo-Saito C, Hodge JW, Kwak H, Kim-Schulze S, Schlom J, et al. (2006) 4$1 \mathrm{BB}$ ligand enhances tumor-specific immunity of poxvirus vaccines. Vaccine 24 : 4975-4986.

30. Abaitua F, Rodriguez JR, Garzon A, Rodriguez D, Esteban M (2006) Improving recombinant MVA immune responses: potentiation of the immune responses to
HIV-1 with MVA and DNA vectors expressing Env and the cytokines IL-12 and IFN-gamma. Virus Res 116: 11-20.

31. Perera LP, Waldmann TA, Mosca JD, Baldwin N, Berzofsky JA, et al. (2007) Development of smallpox vaccine candidates with integrated interleukin-15 that demonstrate superior immunogenicity, efficacy, and safety in mice. J Virol 81: 8774-8783.

32. Chavan R, Marfatia KA, An IC, Garber DA, Feinberg MB (2006) Expression of CCL20 and granulocyte-macrophage colony-stimulating factor, but not Flt3-L, from modified vaccinia virus ankara enhances antiviral cellular and humoral immune responses. J Virol 80: 7676-7687.

33. Symons JA, Tscharke DC, Price N, Smith GL (2002) A study of the vaccinia virus interferon-gamma receptor and its contribution to virus virulence. J Gen Virol 83: 1953-1964

34. Alcami A, Khanna A, Paul NL, Smith GL (1999) Vaccinia virus strains Lister, USSR and Evans express soluble and cell-surface tumour necrosis factor receptors. J Gen Virol 80 (Pt 4): 949-959.

35. Alcami A, Symons JA, Smith GL (2000) The vaccinia virus soluble alpha/beta interferon (IFN) receptor binds to the cell surface and protects cells from the antiviral effects of IFN. J Virol 74: 11230-11239.

36. Alejo A, Ruiz-Arguello MB, Ho Y, Smith VP, Saraiva M, et al. (2006) A chemokine-binding domain in the tumor necrosis factor receptor from variola (smallpox) virus. Proc Natl Acad Sci U S A 103: 5995-6000.

37. Kettle S, Alcami A, Khanna A, Ehret R, Jassoy C, et al. (1997) Vaccinia virus serpin B13R (SPI-2) inhibits interleukin-1beta-converting enzyme and protects virus-infected cells from TNF- and Fas-mediated apoptosis, but does not prevent IL-1beta-induced fever. J Gen Virol 78 (Pt 3): 677-685

38. Kotwal GJ, Isaacs SN, McKenzie R, Frank MM, Moss B (1990) Inhibition of the complement cascade by the major secretory protein of vaccinia virus. Science 250: $827-830$.

39. Bowie A, Kiss-Toth E, Symons JA, Smith GL, Dower SK, et al. (2000) A46R and A52R from vaccinia virus are antagonists of host IL-1 and toll-like receptor signaling. Proc Natl Acad Sci U S A 97: 10162-10167.

40. Alcami A, Smith GL (1992) A soluble receptor for interleukin-1 beta encoded by vaccinia virus: a novel mechanism of virus modulation of the host response to infection. Cell 71: 153-167.

41. Reading PC, Smith GL (2003) Vaccinia virus interleukin-18-binding protein promotes virulence by reducing gamma interferon production and natural killer and T-cell activity. J Virol 77: 9960-9968.

42. Smith VP, Bryant NA, Alcami A (2000) Ectromelia, vaccinia and cowpox viruses encode secreted interleukin-18-binding proteins. J Gen Virol 81: 1223-1230.

43. Clark RH, Kenyon JC, Bartlett NW, Tscharke DC, Smith GL (2006) Deletion of gene A41L enhances vaccinia virus immunogenicity and vaccine efficacy. J Gen Virol 87: 29-38.

44. Moore JB, Smith GL (1992) Steroid hormone synthesis by a vaccinia enzyme: a new type of virus virulence factor. Embo J 11: 1973-1980.

45. Reading PC, Moore JB, Smith GL (2003) Steroid hormone synthesis by vaccinia virus suppresses the inflammatory response to infection. J Exp Med 197: $1269-1278$

46. Stack J, Haga IR, Schroder M, Bartlett NW, Maloney G, et al. (2005) Vaccinia virus protein A46R targets multiple Toll-like-interleukin-1 receptor adaptors and contributes to virulence. J Exp Med 201: 1007-1018.

47. Tscharke DC, Reading PC, Smith GL (2002) Dermal infection with vaccinia virus reveals roles for virus proteins not seen using other inoculation routes. J Gen Virol 83: 1977-1986

48. Froggatt GC, Smith GL, Beard PM (2007) Vaccinia virus gene F3L encodes an intracellular protein that affects the innate immune response. J Gen Virol 88: 1917-1921

49. Chen RA, Jacobs N, Smith GL (2006) Vaccinia virus strain Western Reserve protein B14 is an intracellular virulence factor. J Gen Virol 87: 1451-1458.

50. Roper RL (2006) Characterization of the vaccinia virus A35R protein and its role in virulence. J Virol 80: 306-313.

51. Staib C, Kisling S, Erfle V, Sutter G (2005) Inactivation of the viral interleukin lbeta receptor improves CD8+ T-cell memory responses elicited upon immunization with modified vaccinia virus Ankara. J Gen Virol 86: 1997-2006.

52. Cooray S, Bahar MW, Abrescia NG, McVey CE, Bartlett NW, et al. (2007) Functional and structural studies of the vaccinia virus virulence factor N1 reveal a Bcl-2-like anti-apoptotic protein. J Gen Virol 88: 1656-1666.

53. Domi A, Moss B (2002) Cloning the vaccinia virus genome as a bacterial artificial chromosome in Escherichia coli and recovery of infectious virus in mammalian cells. Proc Natl Acad Sci U S A 99: 12415-12420.

54. Domi A, Moss B (2005) Engineering of a vaccinia virus bacterial artificial chromosome in Escherichia coli by bacteriophage lambda-based recombination. Nat Methods 2: 95-97.

55. Warming S, Costantino N, Court DL, Jenkins NA, Copeland NG (2005) Simple and highly efficient BAC recombineering using galK selection. Nucleic Acids Res 33: e36.

56. Staib C, Drexler I, Ohlmann M, Wintersperger S, Erfle V, et al. (2000) Transient host range selection for genetic engineering of modified vaccinia virus Ankara. Biotechniques 28: 1137-1142, 1144-1136, 1148

57. Staib C, Lowel M, Erfle V, Sutter G (2003) Improved host range selection for recombinant modified vaccinia virus Ankara. Biotechniques 34: 694-696, 698, 700 .

58. Anderson RJ, Hannan CM, Gilbert SC, Laidlaw SM, Sheu EG, et al. (2004 Enhanced CD8+ $\mathrm{T}$ cell immune responses and protection elicited against 
Plasmodium berghei malaria by prime boost immunization regimens using a novel attenuated fowlpox virus. J Immunol 172: 3094-3100.

59. Merchlinsky M, Moss B (1989) Resolution of vaccinia virus DNA concatemer junctions requires late-gene expression. J Virol 63: 1595-1603.

60. Semprini S, Troup TJ, Kotelevtseva N, King K, Davis JR, et al. (2007) Cryptic loxP sites in mammalian genomes: genome-wide distribution and relevance for the efficiency of BAC/PAC recombineering techniques. Nucleic Acids Res 35: $1402-1410$.

61. Tscharke DC, Karupiah G, Zhou J, Palmore T, Irvine KR, et al. (2005) Identification of poxvirus CD8+ $\mathrm{T}$ cell determinants to enable rational design and characterization of smallpox vaccines. J Exp Med 201: 95-104.

62. Tscharke DC, Woo WP, Sakala IG, Sidney J, Sette A, et al. (2006) Poxvirus CD8+ T-cell determinants and cross-reactivity in BALB/c mice. J Virol 80: 6318-6323.

63. Coupar BE, Oke PG, Andrew ME (2000) Insertion sites for recombinant vaccinia virus construction: effects on expression of a foreign protein. J Gen Virol 81: 431-439.

64. Cottingham MG, van Maurik A, Zago M, Newton AT, Anderson RJ, et al. (2006) Different levels of immunogenicity of two strains of Fowlpox virus as recombinant vaccine vectors eliciting $\mathrm{T}$-cell responses in heterologous primeboost vaccination strategies. Clin Vaccine Immunol 13: 747-757.

65. Jarmin SA, Manvell R, Gough RE, Laidlaw SM, Skinner MA (2006) Retention of $1.2 \mathrm{kbp}$ of 'novel' genomic sequence in two European field isolates and some vaccine strains of Fowlpox virus extends open reading frame fpv241. J Gen Virol 87: 3545-3549.
66. Darrah PA, Patel DT, De Luca PM, Lindsay RW, Davey DF, et al. (2007) Multifunctional TH1 cells define a correlate of vaccine-mediated protection against Leishmania major. Nat Med 13: 843-850.

67. Romero P, Maryanski JL, Cordey AS, Corradin G, Nussenzweig RS, et al. (1990) Isolation and characterization of protective cytolytic T cells in a rodent malaria model system. Immunol Lett 25: 27-31.

68. Ma A, Koka R, Burkett P (2006) Diverse functions of IL-2, IL-15, and IL-7 in lymphoid homeostasis. Annu Rev Immunol 24: 657-679.

69. Zimmerli SC, Harari A, Cellerai C, Vallelian F, Bart PA, et al. (2005) HIV-1specific IFN-gamma/IL-2-secreting CD8 T cells support CD4-independent proliferation of HIV-1-specific CD8 T cells. Proc Natl Acad Sci U S A 102: 7239-7244.

70. Falkner FG, Moss B (1990) Transient dominant selection of recombinant vaccinia viruses. J Virol 64: 3108-3111.

71. Kong Y, Yang T, Geller AI (1999) An efficient in vivo recombination cloning procedure for modifying and combining HSV-1 cosmids. J Virol Methods 80: $129-136$.

72. Hartley JL, Temple GF, Brasch MA (2000) DNA cloning using in vitro sitespecific recombination. Genome Res 10: 1788-1795.

73. Smith GL, Mackett M, Moss B (1983) Infectious vaccinia virus recombinants that express hepatitis B virus surface antigen. Nature 302: 490-495.

74. Smith GL, Murphy BR, Moss B (1983) Construction and characterization of an infectious vaccinia virus recombinant that expresses the influenza hemagglutinin gene and induces resistance to influenza virus infection in hamsters. Proc Natl Acad Sci U S A 80: 7155-7159. 